\title{
Analysis of HBT Vector Modulator Phase Shifters Based on Gilbert Cell for sub-THz Regimes
}

\author{
Mohammad Montaseri, Mostafa Jafari-Nokandi, Aarno Pärssinen, and Timo Rahkonen \\ Center for Wireless Communications, Circuits and Systems Lab, ITEE Department \\ University of Oulu, Oulu, Finland \\ Email: \{firstname.lastname\}@oulu.fi
}

\begin{abstract}
This paper concerns with the nonlinearity analysis and compensation of active phase shifters based on vector modulator topologies which utilize Gilbert cell type multipliers. In this respect, both frequency dependence and expansion behavior of such topologies are analyzed. Based on translinear circuits theory, $\log$ /anti-log nonlinear equations are transformed to algebraic linear equations and/or multiplication equations, which facilitates solution for such nonlinear equations. To validate the analyses, a 5-bit active phase shifter was designed and simulated in $0.13 \mathrm{um}$ HBT technology node. 3D electromagnetic simulation results verify the consistency of the analysis and proposed compensation technique.
\end{abstract}

Keywords-HBT, active phase shifter, vector modulator, Gilbert cell, translinear analysis, sub-THz, 5G, 6G

\section{INTRODUCTION}

Beamforming offers several advantages to the new wireless systems; the most important of which is, it enables lower transmit-power per unit block in phased array applications when the link-budget is concerned. It also facilitates multipath links in congested line-of-sight (LOS) link. Spatial scanning capability of such systems empowers the multi-user multi-input-multi-output (MIMO) systems including $5 \mathrm{G} / 6 \mathrm{G} / \mathrm{IoT}[1]$. On the other hand, the electronic steerable directional antenna patterns offered by such systems has improved radar and imaging applications as well [2]. Phase shifters (PS) are the key enablers that facilitate such broad applications. There are several different types of PS topologies all of which may be categorized into two major classes namely, "passive" and "active" PS's. The most important advantage of the passive PS's is their linearity while they suffer mostly from higher losses and limited phase resolution/variation [2] - [6]. Active PS's, on the other hand, offer gain as well as much better/wider phase resolution/variation at the cost of nonlinearity of the active devices [1], [7] - [17]. Vector modulators are dominantly utilized to shift the phase in active PS's. Thus, vector modulators play a critical role in all the abovementioned systems.

As the Gilbert cell structure offers reduced circuit complexity, it is possible to implement it at $6 \mathrm{G}$ candidate frequencies, i.e. sub-THz, bands. Moreover, its importance in nearly every in-phase/quadrature (I/Q) mixer, multiplier, variable gain amplifier (VGA), has made it a key building block of the mentioned systems.

In order to model the nonlinearities of active phase shifters based on Gilbert cell, translinear circuit analysis method has been applied to express the log/anti-Log equations governing the circuit operation [18]. In this respect the scheme of vector modulator PS's along with their operating principals is explained in section II. Section III concerns with the operating principles of Gilbert cells based on translinear circuit analysis

This work was supported by the Academy of Finland 6Genesis Flagship (grant no. 318927). theory. The origin of nonlinear behavior of the mentioned phase shifter circuits is analyzed in section IV. The frequency dependent phase error is also covered in the same section. Simulation results are then presented in section V.

\section{Vector MOdUlator Phase ShIFTERS}

\section{A. Block Diagram}

Fig. 1 illustrates the block diagram of the vector modulator PS's concept, where the input signal is decomposed into I and $\mathrm{Q}$ vector components and applied to the VGAs. Based on the requirements, the I and Q vector signals are weighted by the VGAs and the resultant weighted vectors are then added to construct the phase shifted version of the original input signal.

\section{B. Circuit Level Implementation of the Blocks}

The quadrature signal generation (Fig. 1) may be implemented in several various circuit schemes such as poly phase filters [11], hybrid couplers [2], [12], lumped elements [13], [14]. All the mentioned implementations share the same property of being passive in nature, which are hardly ever sources of nonlinearity; unlike the VGAs case.

There are also different circuit topologies for the VGA stages, e.g. programmable differential amplifiers [1], [17], Gilbert cell [15], etc.; all of which utilize differential amplifiers circuitry as the core analog signal processor of the vector modulator PS's. The presence of transistors, certainly, contribute to the nonlinearity, hence improper constellation point relocation in either compression or expansion manner is inevitable. Thus, the rest of this paper is devoted to HBT differential pair and Gilbert cell analysis.

\section{I/Q AMPLITUDE WEIGHTING CIRCUITRY}

As mentioned earlier, comprised of $T_{1}-T_{6}$, Gilbert cell has been exploited for the VGAs (Fig. 2). $T_{7}$ and $T_{8}$ have been utilized to linearize the tanh property of the $i-v$ characteristic of the differential pair [19]. With I and Q branches sharing the same circuit scheme, Fig. 2 summarizes the whole vector modulator. $C_{b}$ decouples the RF signal from the control circuitry, thus the $T_{7}$ and $T_{8}$ carry only DC currents.

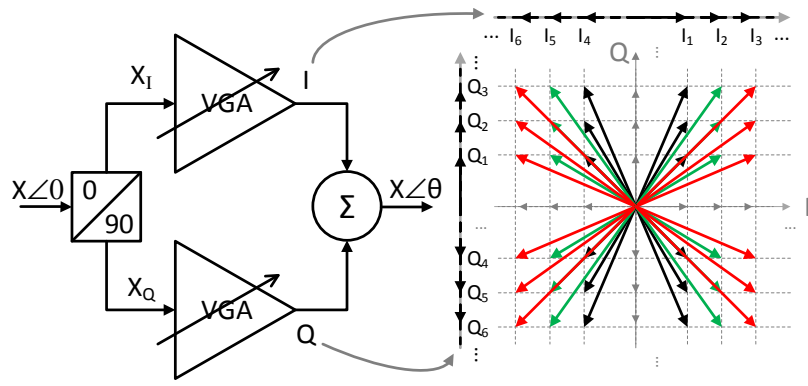

Fig. 1. Block diagram of the vector modulator phase shifter along with the weighted I/Q componenst and the resultant shifted version of the input signal. 
The generated I/Q signals are applied to the tail transistors of the Gilbert cells, i.e. $T_{5}$ and $T_{6}$, and based on the control level, the current steered towards either of the transistors of the differential pairs, i.e. $T_{1}-T_{4}$, where the I/Q vector signals are weighted. Finally, the weighted vector signals are superposed to form the phase shifted version of the input signal at the $\mathrm{I}^{+}$and $\mathrm{I}^{-}$output nodes.

\section{TRANSLINEAR ANALYSIS}

Based on the translinear (TL) analysis method [18], the algebraic sum of all $V_{B E}$ 's in an even numbered BJTs in a loop translates to the multiplication of the corresponding clock wise collector currents to be equal to those in counter clock wise. This is shown in Fig. 3 and formulated as (1) and (2).

$$
\begin{gathered}
\sum_{i=1}^{N} V_{B E_{2 i}}=\sum_{i=1}^{N} V_{B E_{2 i-1}} . \\
\prod_{i=1}^{N} I_{2 i}=\prod_{i=1}^{N} I_{2 i-1} .
\end{gathered}
$$

The constellation points of a phase shifter based on Gilbert cell can be calculated according to (1) and (2). To do so, the whole vector modulator is divided to primary loops shown in Fig. 4, in which $I_{C t r l}{ }^{ \pm}, I_{R F}$, and $I_{1}^{\prime+}$ and $I_{1}^{\prime-}$ are DC control current, RF tail current produced by either of the tail transistors, and the output currents of the differential pair, respectively. Thus, we have, $I_{1}^{\prime+} I_{7}=I_{1}^{\prime-} I_{8}$ and $I_{R F}=I_{1}^{\prime+}+$ $I_{1}^{\prime-}$. With the definition of $I_{C t r l}^{+}=X I$ and $I_{C t r l}^{-}=(1-X) I$, the output currents of interest can be defined as

$$
\begin{gathered}
I_{1}^{\prime+}=(1-X) I_{R F} \\
I_{1}^{\prime-}=X I_{R F} .
\end{gathered}
$$

wherein $0 \leq X \leq 1$. By $\mathrm{KCL}$, the currents $I_{I}^{+}=I_{1}^{\prime+}+I_{2}^{\prime-}$, $I_{I}^{-}=I_{1}^{\prime-}+I_{2}^{\prime+}, I_{Q}^{+}=j\left(I_{1}^{\prime+}+I_{2}^{\prime-}\right)$, and $I_{Q}^{-}=j\left(I_{1}^{\prime-}+I_{2}^{\prime+}\right)$ can be obtained as follows.

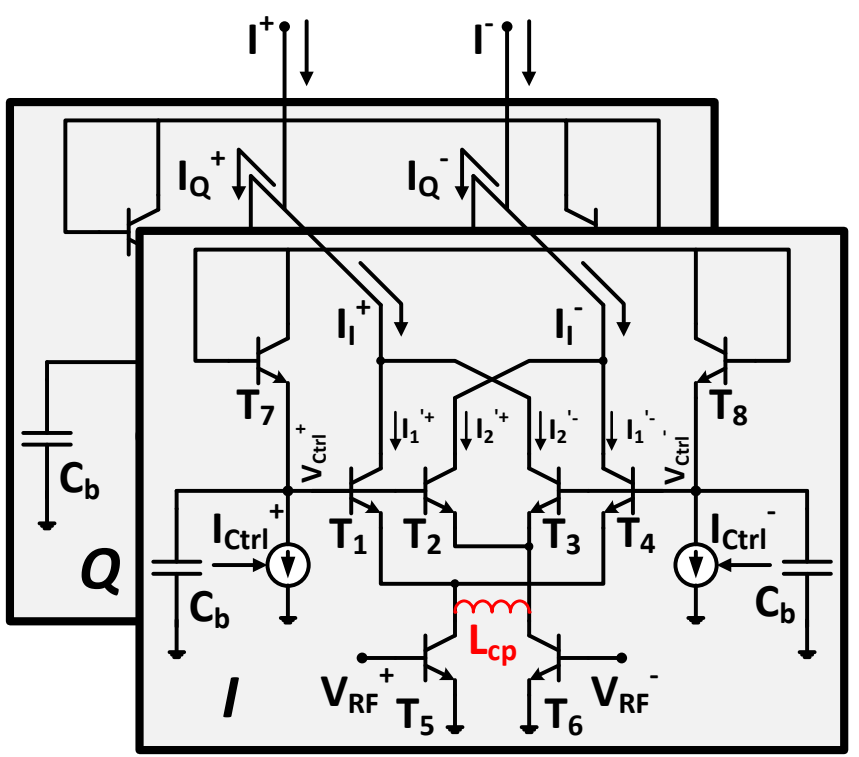

Fig. 2. The whole $\mathrm{I} / \mathrm{Q}$ processing circuitry based on current summation mechanism

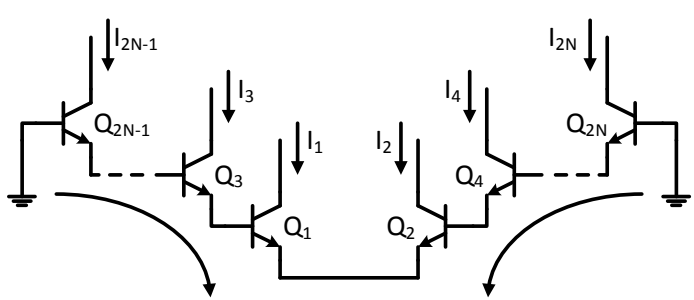

Fig. 3. A typical single loop translinear BJT circuit.

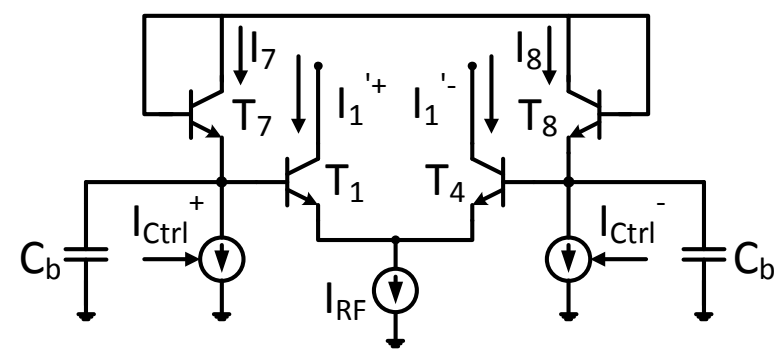

Fig. 4. A fundamental TL loop in a Gilbert cell.

$$
\begin{gathered}
I_{I}^{+}=-I_{I}^{-}=(1-2 X) I_{R F} \\
I_{Q}^{+}=-I_{Q}^{-}=j(1-2 Y) I_{R F} .
\end{gathered}
$$

The notations " $j$ " and " $Y$ " in (6) corresponds to a $90^{\circ}$ phase rotation due to the quadrature nature of the input signals to the vector modulator and separate control circuitry for the Q branch of the overall vector modulator circuit, respectively. With that in mind, and the definition of $I^{+}=I_{I}^{+}+I_{Q}^{+}$and $I^{-}=I_{I}^{-}+I_{Q}^{-}$, the overall differential output current is defined as

$$
I=I^{+}-I^{-}=2 I_{R F}((1-2 X)+j(1-2 Y))
$$

where $X, Y \in[0,1]$ are control-to-phase shift coefficients. Equation (7) predicts the behavior of the vector modulator phase shifters based on Gilbert cell (Fig. 2). This holds valid up to frequencies well below the unity current gain frequency, i.e. $f_{t}$, of the transistors. This however is not the case when the operating frequency approaches the $f_{t}$, as the high order parasitic elements start to pose performance degradation to the circuit. The most dominant frequency dependent parasitic element of a BJT is its base-emitter junction capacitance, i.e. $C_{\pi}$. Thus, (3) - (7) must be rewritten with respect to the impact of $C_{\pi}$. It should be noted that, due to the presence of $C_{b}$, the control circuitry remains in DC domain. Thus,

$$
\begin{gathered}
I_{1}^{\prime+} I_{7}=I_{1}^{\prime-} I_{8} \Rightarrow I_{1}^{\prime-}=I_{1}^{\prime+} \times \frac{I_{7}}{I_{8}}=I_{1} \times \frac{X}{1-X} \\
I_{R F}=I_{1}^{\prime+}+I_{1}^{\prime-}+C_{\pi} V_{T}\left(\frac{1}{I_{1}^{\prime+}} \frac{\partial}{\partial t} I_{1}^{\prime+}+\frac{1}{I_{1}^{\prime \prime}} \frac{\partial}{\partial t} I_{1}^{\prime-}\right) .
\end{gathered}
$$

Equation (8) is a differential equation of type "Bernoulli", the solution of which to a sinusoidal excitation, i.e. $I_{R F}=I_{m}(1+\cos \omega t)$, is of the form, 


$$
\left(I_{1}^{\prime+}\right)^{-1}=\underbrace{e^{-\frac{I_{m} \sin \omega t}{2 \omega C_{\pi} V_{T}}}}_{i}(\frac{1}{2 C_{\pi} V_{T}(1-X)} \underbrace{\int e^{\frac{I_{m} \sin \omega t}{2 \omega C_{\pi} V_{T}}} d t}_{i i}+C_{o})
$$

$C_{o}$ in (9) is a constant which can be defined with respect to initial conditions of the vector modulator circuit. To calculate (9), the terms $i$ and $i i$, can be expanded in a Fourier series, in which both terms are expressed by the definition of modified Bessel function of order $\mathrm{n}$ and argument $\left(I_{m} / 2 \omega C_{\pi} V_{T}\right)$. Since the operating frequency is quite close to $f_{t}$, only the fundamental harmony may be of interest, that is the order of the Bessel function can be limited to $n=1$. Thus,

$$
I_{1}^{\prime+} \propto(1-X) \underbrace{\left(J_{0}(x)+2 \omega C_{\pi} V_{T} J_{1}(x)\right)}_{\text {expansive term }} \sin \omega t .
$$

Where $J_{0}$ and $J_{1}$ are the Bessel functions of the zeroth and first order respectively and $\mathrm{x}$ is the argument of the Bessel function defined as $\left(x=I_{m} / 2 \omega C_{\pi} V_{T}\right)$.

Equation (7) must be modified in accordance with (10); i.e. all the terms need to be replaced with their corresponding term in (10). Equation (10) approximates to the sub-THz behavioral model of vector modulator PS's that predicts the impact of the parasitic capacitance $C_{\pi}$ at different frequencies. Due to the presence of modified Bessel function, the nonlinear mapping of the constellation points in an expansive manner is expected. It is also clear that as the operating frequency approaches zero (10) approaches (5) and (6) and hence (7).

Part of such nonlinearities can be relieved by inductive compensation in the TL loop in proximity of the base-emitter junction capacitances. Since there exist two coupled loops, the inductance element, i.e. $L_{c p}$, can be shared between the loops shown in Fig. 2. The approximate value of $L_{c p}$ can be determined by the help of Fig. 5 as

$$
L_{c p}=\frac{1}{\omega^{2} C_{\pi}}
$$

In order to dimension $L_{c p}$, the parasitic capacitances present in each primary TL loop of Fig. 2 were placed in Fig. 5. This is starting from collector of $\mathrm{T}_{5}$, i.e. $\mathrm{T}_{5 \mathrm{C}}$, to the base of $\mathrm{T}_{1}$, i.e. $\mathrm{T}_{1 \mathrm{~B}}$, where parasitic capacitance of the control circuitry, i.e. $\mathrm{C}_{\mathrm{C}}$, is added to the node, and then to $\mathrm{T}_{7 \mathrm{~B}}$ and $\mathrm{T}_{4 \mathrm{~B}}$ and back to the $\mathrm{T}_{5 \mathrm{C}}$. By the same token, other parasitic capacitances may be added to form Fig. 5 and from that the equivalent parasitic capacitance can be approximately estimated.

\section{Simulation Results}

In order to verify the proposed compensation method and validation of the extracted equations, a phase shifter was designed which utilizes Gilbert cell, as the core signal processing circuitry, and a 5-bit current DAC, for the sake of level control and constellation point mapping. The circuit was designed in HBT $0.13 \mathrm{um}$ technology node with $f_{t} / f_{\max }$ of $350 / 550 \mathrm{GHz}$. In order to track the impact of frequency variations on constellation points, the operating frequency was swept from frequencies much below $f_{t}$ up to those quite close to $f_{t}$. The feasibility of the proposed compensation method is examined at $300 \mathrm{GHz}$. To account for the impact of the metallic parts, a 3D EM simulation was performed. A simplified Gilbert cell structure is shown in Fig. 6.
Fig. 7 illustrates the simulation result of the designed circuit at $10 \mathrm{GHz}$. It can be seen at low frequencies the response of the phase shift to the control coefficients is totally linear with neither sign of expansion nor constellation points curvature mapping. This is due to that fact that parasitic capacitances have a minor impact at such frequency bands.

Increasing the operating frequency up to $100 \mathrm{GHz}$ will demonstrate the first symptoms of the constellation mapping deterioration. This is shown in Fig. 8. It is immediately seen that the constellation points not only have rotated but also expanded over the control range. This is because the parasitic reactive components start to manifest, i.e. $\omega C_{\pi}$ values are comparable to $g_{m}$.

With increasing operating frequency and approaching the $f_{t}$ of the transistors, the situation tends to get worse. This is quite compatible with what was foreseen by (10). Drastic expansion nonlinear mapping is clearly observed in the constellation point (see Fig. 9).

After applying the proposed compensation method, the nonlinear behavior gets relieved to some extent (see Fig. 10). However, a closer investigation of the constellation points illustrates existence of some nonlinearity (Fig. 11). This is because the proposed compensation technique, compensates mostly the linear average part of the impact of the parasitic capacitances even though they are nonlinear in their nature.

The state trajectory of the transconductance of the transistors at different frequency bands is also very informative. As can be seen from Fig. 12, at frequencies well below $f_{t}$, e.g. $10 \mathrm{GHz}$, the transconductance state trajectories hardly show any nonlinear behavior. Nonlinearities as well as drastic phase rotation increase with operating frequency. This is shown in Fig. 13. By the virtue of the proposed compensation method, part of the phase rotation as well as nonlinearities of the state trajectories are averaged out.

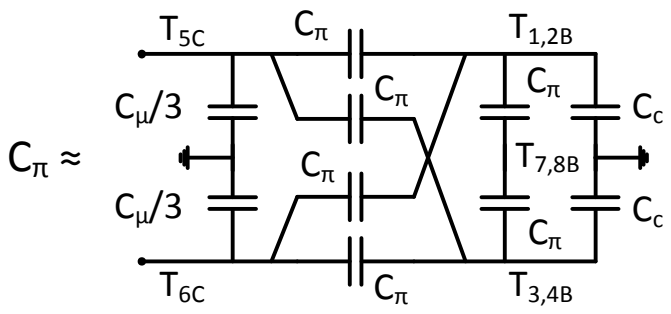

Fig. 5. Parasitic capacitances and their equivalence to be compensated by $L_{c p}$.

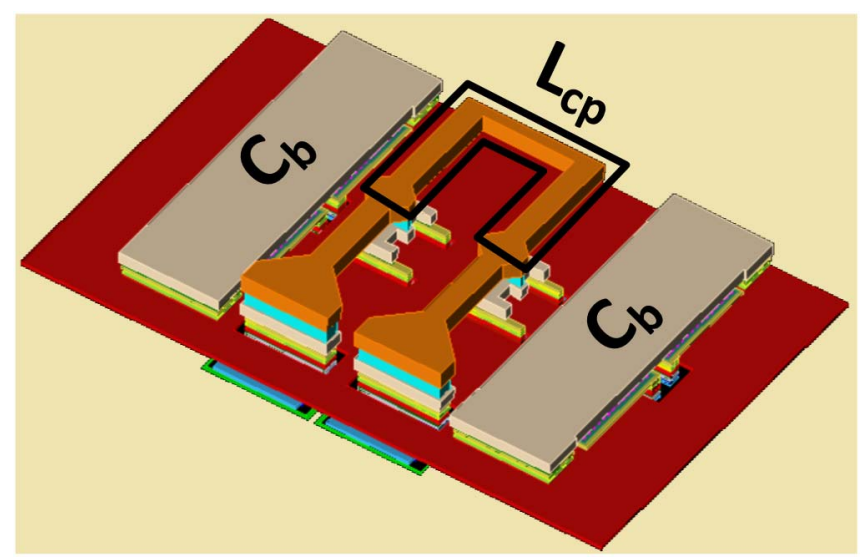

Fig. 6. A simplfied 3D view of the Gilbert cell utilized in the design. 


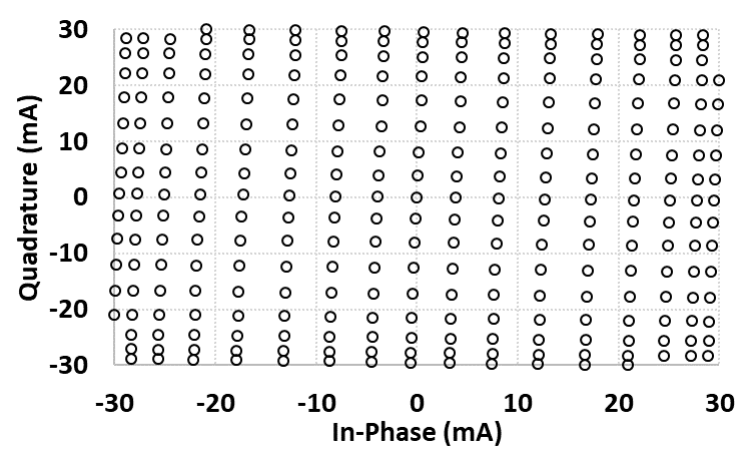

Fig. 7. Constellation points at low frequencies.

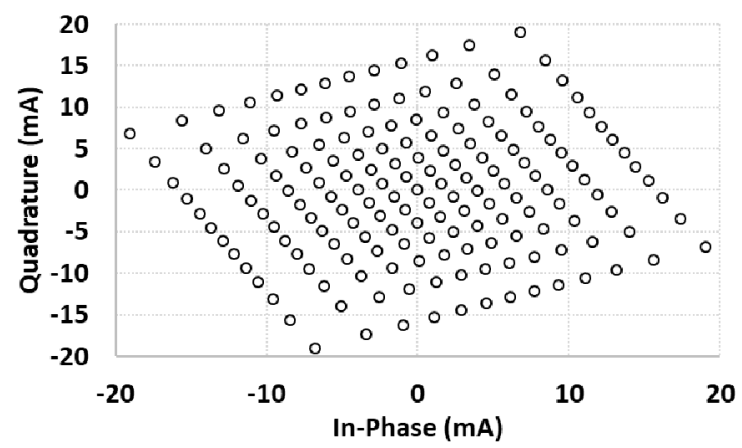

Fig. 8. The signes of constellation deterioration as the frequency increases.

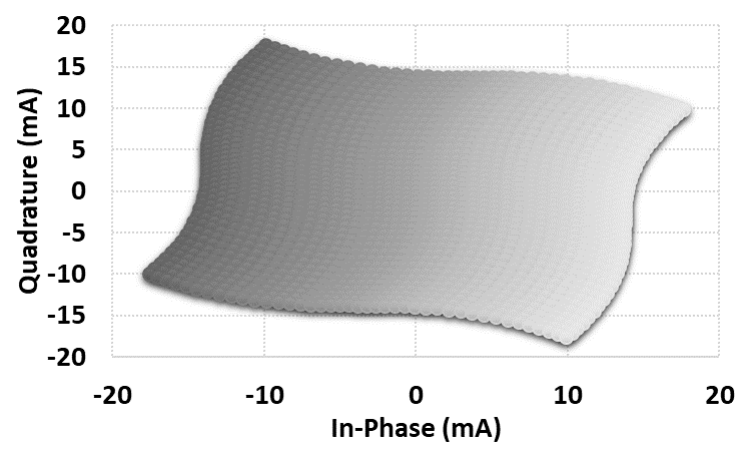

Fig. 9. Total constellation point deterioration at frequencies close to $f_{t}$ of the transistors, before compensation.. The operating frequency if $300 \mathrm{GHz}$.

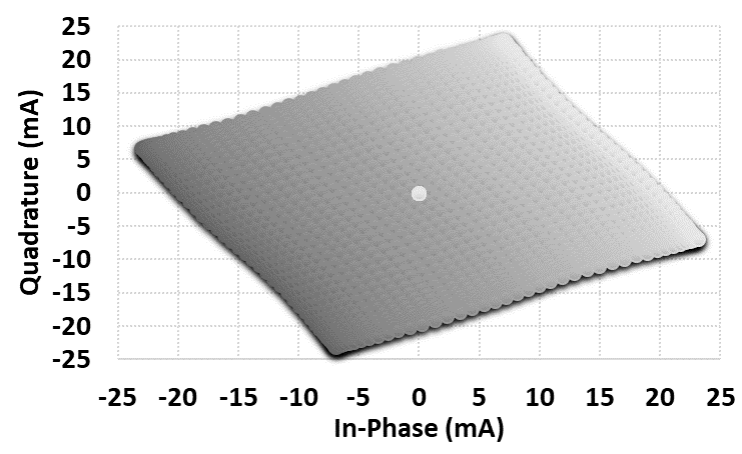

Fig. 10. The impact of the proposed compensation technique on the constellation points at $300 \mathrm{GHz}$.

\section{CONCLUSION}

Active phase shifters based on Gilbert cell were analyzed in this paper. Nonlinearity mechanism of such circuits was determined and approximately modeled. Based on the analysis the origin of the nonlinearity was recognized. A compensation method was proposed and validated based on simulations which proved the concept. To support the simulations, the transconductance state trajectories were presented to illustrate phase rotation and nonlinear distribution of the constellation points.

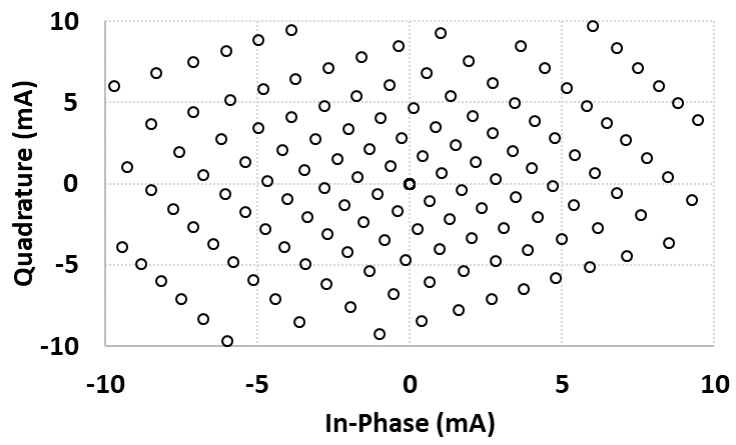

Fig. 11. Magnified constellation points of the compensated PS.

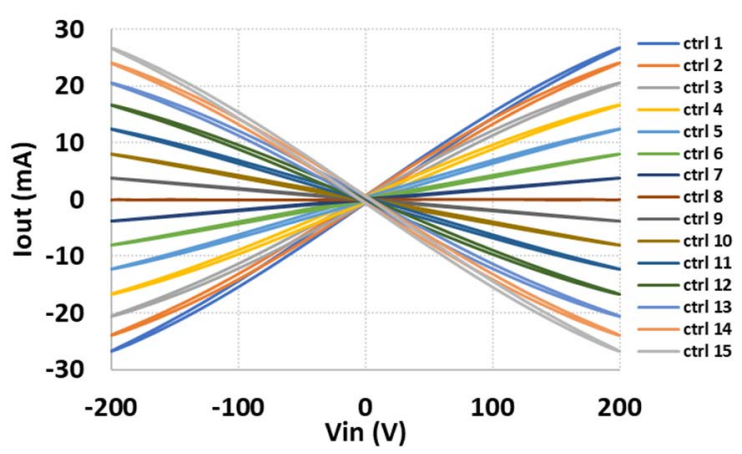

Fig. 12. State trajectory of the IV of the Gilbert cell at $10 \mathrm{GHz}$.

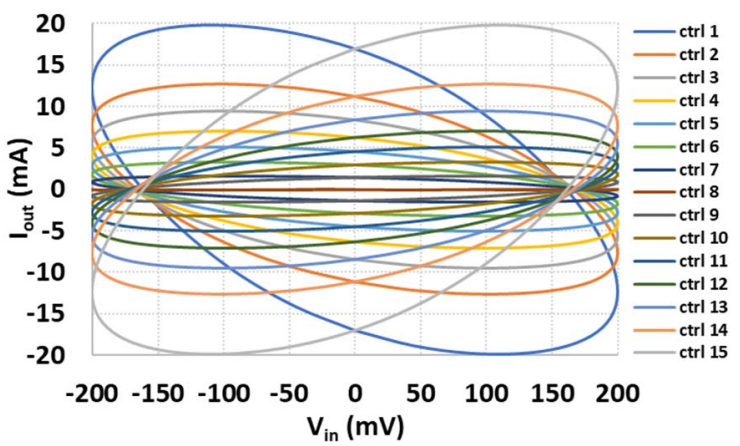

Fig. 13. Phase rotation and nonlinearity of the state trajectory of the IV of the Gilbert cell at $300 \mathrm{GHz}$, before compensation.

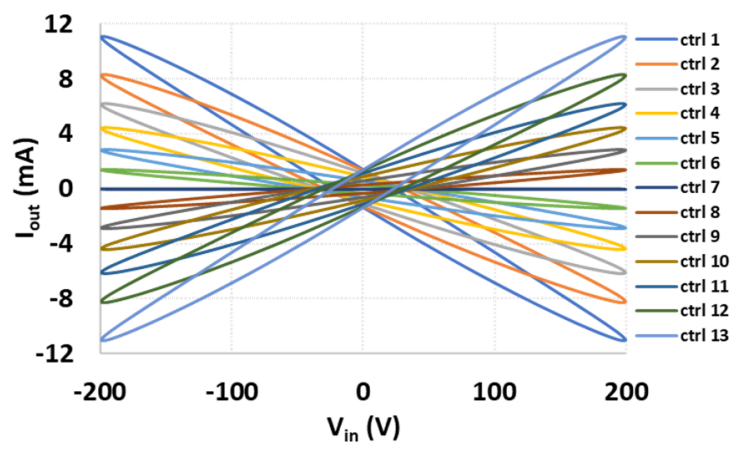

Fig. 14. Phase rotation and nonlinearity of the state trajectory of the IV of the Gilbert cell at $300 \mathrm{GHz}$, after compensation. 


\section{REFERENCES}

[1] G. Eason, B. Noble, and I. N. Sneddon, "Two mm-wave vector modulator active phase shifters with novel IQ generator in $28 \mathrm{~nm}$ FDSOI CMOS," IEEE J. Solid State Circuits, vol. 52, NO. 2, pp. 344 356, Feb 2017.

[2] H. Krishnaswamy, A. Valdes-Garcia, and JW. Lai, "A silicon-based, all-passive, $60 \mathrm{GHz}$, 4-element, phased-array beamformer featuring a differential, reflection-type phase shifter," IEEE International Symposium on Phased Array Systems and Technology, Oct. 2010.

[3] J. Staudinger, "Delay line phase shifter with selectable phase shift," U.S. Patent 2013 0,194,017 A1, Aug. 1, 2013.

[4] A. Valdes-Garcia et al., "A fully integrated 16-element phased-array transmitter in SiGe BiCMOS for 60-GHz communications," IEEE J. Solid-State Circuits, vol. 45, no. 12, pp. 2757-2773, Dec. 2010.

[5] Y. Yu, P. G. M. Baltus, A. De Graauw, E. van der Heijden, C. S. Vaucher, and A. H. M. van Roermund, "A $60 \mathrm{GHz}$ phase shifter integrated with LNA and PA in $65 \mathrm{~nm}$ CMOS for phased array systems," IEEE J. Solid- State Circuits, vol. 45, no. 9, pp. 1697-1709, Sep. 2010.

[6] B.-W. Min and G. M. Rebeiz, "Single-ended and differential Ka-band BiCMOS phased array front-ends," IEEE J. Solid-State Circuits, vol. 43, no. 10, pp. 2239-2250, Oct. 2008.

[7] D. Pepe and D. Zito, "A 78.8-92.8 GHz 4-bit 0-360 ${ }^{\circ}$ active phase shifter in $28 \mathrm{~nm}$ FDSOI CMOS with $2.3 \mathrm{~dB}$ average peak gain," in Proc. 41 st Eur. Solid-State Circuits Conf. (ESSCIRC), Graz, Austria, 2015, pp. 64-67.

[8] C.-Y. Kim, D.-W. Kang, and G. M. Rebeiz, "A 44-46-GHz 16-element SiGe BiCMOS high-linearity transmit/receive phased array," IEEE Trans. Microw. Theory Techn., vol. 60, no. 3, pp. 730-742, Mar. 2012.

[9] L. O. Mereni, D. Pepe, and D. Zito, "Analyses and design of 95-GHz SoC CMOS radiometers for passive body imaging," Anal. Integr. Circuits Signal Process., vol. 77, no. 3, pp. 373-383, Dec. 2013.
[10] K.-J. Koh, J. W. May, and G. B. Rebeiz, “A millimeter-wave (40-45 $\mathrm{GHz}$ ) 16-element phased-array transmitter in 0.18- $\mu \mathrm{m}$ SiGe BiCMOS technology," IEEE J. Solid-State Circuits, vol. 44, no. 5, pp. 14981509, May 2009.

[11] S. Kulkarni, D. Zhao, and P. Reynaert, "Design of an optimal layout polyphase filter for millimeter-wave quadrature LO generation," IEEE TCAS II, vol. 60, NO. 4, pp. 202-206, April 2013.

[12] N. Mazor, O. Katz, R. Ben-Yishay, D. Liu, A. Valdes Garcia, D. Elad, "SiGe based Ka-band reflection type phase shifter for integrated phased array transceivers," IEEE MTT-s IMS, May 2016.

[13] Tso-Wei Li, and Hua Wang, "A millimeter-wave fully integrated passive reflection-type phase shifter with transformer-based multiresonance loads for $360^{\circ}$ phase shifting," IEEE TCAS-I, vol. 65, no. 4, pp. 1406-1419, April 2018.

[14] H. Zarei, C.T. Charles, and D.J. Allstot, "Reflective-type phase shifters for multiple-antenna transceivers," IEEE TCAS-I, vol. 54, no. 8, pp. 1647-1656, Aug. 2007.

[15] KJ. Koh, J.W. May, and G.M. Rebeiz, "A Q-band (40-45 GHz) 16element phased-array transmitter in $0.18-\mu \mathrm{m}$ SiGe BiCMOS technology," IEEE Radio Frequency Integrated Circuits Symposium, June 2008.

[16] K. Kibaroglu, M. Sayginer, and G.M. Rebeiz, "A low-cost scalable 32element $28-\mathrm{GHz}$ phased array transceiver for $5 \mathrm{G}$ communication links based on a $2 \times 2$ beamformer flip-chip unit cell," IEEE J. solid-state circuits, vol. 53, no. 5, pp. 1260-1274, May 2018.

[17] Y. Yeh, B. Walker, E. Balboni, and B. Floyd, "A 28-GHz Phased-Array Receiver Front End With Dual-Vector Distributed Beamforming", IEEE J. Solid-State Circuits, vol. 52, no. 5, pp. 1230-1244, May 2017

[18] B. Gilbert, "Translinear circuits: a proposed classification," Electronics Letters, vol. 11, no. 1, pp. 14 - 16, Jan. 1975.

[19] P.R. Gray, P.J. Hurst, S.H. Lewis, R.G. Meyer, Analysis and Design of Analog Integrated Circuits, $5^{\text {th }}$ ed., John Wiley, 2009. 